\title{
Optimal Policy for the Replacement of Industrial Systems Subject to Technological Obsolescence - Using Genetic Algorithm
}

\author{
Mohamed Arezki Mellal ${ }^{1}$, Smail Adjerid ${ }^{1}$, Djamel Benazzouz ${ }^{1}$, \\ Sofiane Berrazouane ${ }^{2}$, Edward J. Williams ${ }^{3}$ \\ 1 LMSS, Faculty of Engineering Sciences (FSI), M'Hamed Bougara University, \\ Avenue de l'Indépendance, 35000 Boumerdes, Algeria \\ mellal-mohamed@umbb.dz, adjerid_s@umbb.dz,dbenazzouz@umbb.dz \\ 2 Faculty of Engineering Sciences (FSI), M'Hamed Bougara University \\ Avenue de l'Indépendance, 35000 Boumerdes, Algeria \\ berrazouane_so@umbb.dz \\ 3 College of Business, Decision Sciences, University of Michigan \\ 4901 Evergreen Road, Dearborn, 48126 Michigan, USA \\ williame@umd.umich.edu
}

\begin{abstract}
The technological obsolescence of industrial systems is characterized by the existence of challenger units possessing identical functionalities but with improved performance. This paper aims to define a new approach that makes it possible to obtain the optimal number of obsolete industrial systems which should be replaced by new-type units. This approach presents a new point of view compared with previous works available in the literature. The main idea and the originality of our approach is that we apply a genetic algorithm (GA) by considering the failure frequency, the influence of the environment/safety factors of the old-type systems and the purchase/implementation cost of the new-type units. These parameters are introduced in order to optimize this type of replacement in the context of engineering.
\end{abstract}

Keywords: technological obsolescence; industrial systems; replacement policy; failure frequency; safety/environment factors; genetic algorithm (GA)

\section{Introduction}

Often the behavior analysis of industrial systems in engineering is based on the study of monitoring and diagnostics, but technological obsolescence is neglected in the models. Nowadays, technological change is abrupt and the great majority of industrial systems are subject to obsolescence. An item becomes obsolete when a 
new-type unit is available and performs the same functionalities but with improved performance. Hence, the necessity of an approach in order to deal with technological obsolescence is important for industrial firms. In most previous works devoted to the study of the dependability of industrial systems, the authors have not taken into consideration technological change.

In [1-4], several industrial plants were studied in order to achieve models of dependable installations, but the influence of technological obsolescence has not been considered in the approaches.

Technological improvement has an impact on the life-cycle of the industrial plants due to the unavailability of spare parts, and thus, if this problem is not considered, it will generate random and various consequences, such as accidents, stoppage of production, environmental disaster, etc.

The improvement in performance of the new technology items can be understood as smaller failure rates, lower pollution, more security, lower energy consumption, etc. At the same time, it is difficult to determine the optimal policy for the replacement of old technology units by new ones in the context of engineering (dependability study) and not only in the context of economics. On the other hand, it is economically more interesting for industrial firms to replace the old type units gradually to benefit from their residual lifetime. Most often, the authors studied the technological obsolescence under two contexts: the first one is strictly based on economic assumptions and other parameters are neglected (e.g., [5-7]). The second one aims to define an approach that takes into account various parameters of engineering and economic elements, such as failure frequencies, costs and strategy of maintenance, reliability, etc. We can cite [8-11].

The aim of our work is therefore to define a replacement policy of these obsolete industrial systems in the context of engineering and to help the decision maker find the optimal systems which should be replaced among them.

This paper summarizes and extends the works presented in previous papers. It is organized as follows: Section 2 describes a brief literature review of previous works, the assumptions on which they were based, and an overview. Section 3 illustrates our approach and the assumptions on which it is based, and we conclude this section with a case study and numerical results. Finally, we conclude this paper by suggesting some possible perspectives and extensions of our approach.

\section{Thematic Review of the Literature}

Several researchers have been studying the problem of technological obsolescence in industry from many points of view. The first paper was published by Elton and Gruber [5] in 1976. Their work considered one single component characterized by an annual income, purchase cost, resale value, which decreases with the age of the 
component, and an aging factor, which reduces the income. Technological change generates efficiency which was given in the model by an increase of an annual income of the new-technology unit with a factor denoted $g$. The failures were not considered, but the age of the component was modeled by a linear decrease of the income factor, $h$, generated over time. The model was given as follows:

$r T+e^{-r T}=1+\frac{r^{2}(I-S)}{(g+h)-r s}$

where $r$ is the discount rate for a period $t, I$ is the purchase cost of the oldgeneration component, $S$ is the purchase cost of the new-type unit, $s$ is the resale value per time unit of the operating unit and $T$ is the time interval for a component replacement. The authors of this work considered that the strategy which maximizes the income consists of replacing the component at regular interval $T$, where $T$ is the solution of (1).

In [6], the authors considered one component subject to technological obsolescence. The model was proposed for a discretized time of replacement at the appearance of the new-type unit without taking into account the failure rates.

In [7], the authors considered a geometric technological change of several industrial systems and they solved a continuous-time optimization problem to define an optimal replacement policy by searching for the optimum of (3).

$$
\begin{aligned}
& L(t)=\int_{d(t)}^{t} m(\tau) d \tau \\
& \min I=\int_{0}^{T} e^{-r t}\left(\int_{d(t)}^{t} q(\tau, t) m(\tau) d \tau+p(t) m(t)\right) d t
\end{aligned}
$$

where $L(t)$ is the number of systems in service, $a(t)$ is the installation time of the obsolete systems replaced at time $t, m(t)$ is the current investment (the number of new installed systems), $q(\tau, t)$ is the specific maintenance cost of the vintage $\tau$ at time $t, p(t)$ is the purchase price and installation cost of a new system, and $r$ is the discounting factor, $r>0$. Then $t-a(t)$ is the lifetime of the industrial plant (the age of the oldest system still in use). The constraint of (3) was given as follows:

$0 \leq m(t) \leq M(t), d(t) \leq t$

where $M(t)$ is the number of the old-type units. The authors of this article neglected the failure rates and other paramount parameters.

In [8], the case of one single industrial item subject to aging and technological obsolescence was proposed in the model. The authors assumed that the time of the first failure of the component follows a Weibull distribution with two parameters. Several maintenances are undertaken at regular intervals and the repairs are considered. They assumed that the maintenance resets the component to the same 
status at the beginning of the maintenance interval. The authors modeled the probability of failure of the component using a constant failure rate per part. The constant failure rate was given as follows:

$$
\lambda^{*}=\left(\frac{1}{\alpha}\right)^{\beta} T^{\beta-1}
$$

where $\alpha$ is the scale parameter, $\beta$ is the shape parameter and $T^{\beta-1}$ is the maintenance interval. The authors assumed that the increase in this failure rate is due to the aging of the component and they formalized the issue of obsolescence in a quantifiable manner. To account for the various issues at stake, they postulated that as calendar time goes by new components are available on the market, and they are characterized by a failure rate which decreases exponentially. The authors concluded that this component in service can be either periodically maintained or preventively replaced by a new-type unit. They solved the problem by assessing the costs using Monte Carlo simulations.

In [11], a repairable system that operates continuously to the degraded state was studied. The model was presented by the following decisions about the interventions: do nothing, maintain or to replace by a new-type unit.

In $[9,10,12,13]$, the authors studied the following case: A set of $N$ identical and independent industrial items.

The authors of [9] proposed that these components can be either preventively or correctively replaced by new-type units and the replacements take negligible time.

The works of Elmakis et al. [13] are characterized by this assumption: the failure rate $\lambda_{0}$ of each component is constant. The proposed approach in their paper is called the " $K$ strategy" (Fig. 1 ) and it is based as follows: first, new-type components are used only to replace failed old-type units; then, after $K$ corrective actions of this kind, the $N-K$ old-type remaining components are preventively replaced by new-type ones at the time of the $K^{\text {th }}$ corrective intervention.

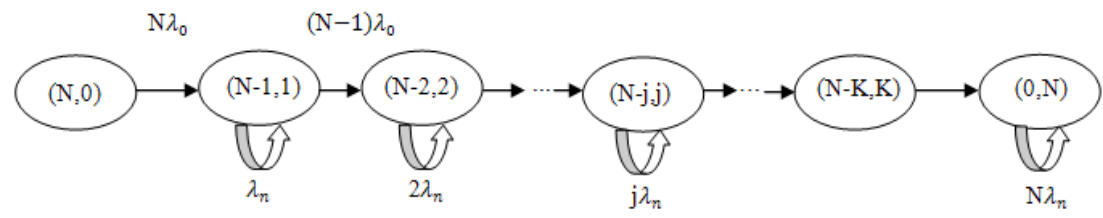

Figure 1

Diagram of $K$ strategy

The " 0 " strategy represents the preventive replacement of all old-type components at the initial moment. To determine the value of $K$, the authors proposed a Monte Carlo simulation to assess the costs generated by each value of $K$.

In $[10,12,14]$, the authors proposed extensions of the " $K$ strategy" by taking into consideration the failure frequencies as a Weibull law of this form. 
In the contribution of the works presented in [10], the authors proposed a model for $N$ identical components, but with several challengers. A probability of incompatibility was accounted to deal with the fact that the on-site implementation of new-type units could turn out to be problematic, and some replacements could not be immediately successful, as operators have no experience to rapidly implement the new-technology unit. In [15], a test case was performed using Petri nets to model the different replacement strategies proposed in [10].

All the works cited in this section proposed models without taking into account the influence of the old-system units on the environment and the safety criterion. These factors are recommended to be considered in the model, especially nowadays with the environmental problems and the industrial accidents.

\section{Model Description}

In this paper, we introduce a more realistic approach compared with the works illustrated in Section 2.

The case studied in our work is the following: A set of $N$ different and independent obsolete industrial systems, one challenger per old-type unit is available, the industrial firm devotes a special budget to deal with technological obsolescence at the end of the year and the transition between the generations of the units will be done. To study the transition problem, we consider the following important data:

- Failure frequency per hour during the year of each old-type unit.

- An annual budget is intended to overcome the technical-economic impact of technological obsolescence.

- The purchase and implementation cost of each new-type unit (challenger) is fixed.

- We select only the compatible challenger for the replacement to avoid production delays.

- Each old-type unit is characterized by its environment and safety factors which vary in the scale $[0,1]$ (where a $100 \%$ non-polluting and secure system is assigned the value 1 ).

The data of each system are summarized as follows:

$$
\text { System }_{n}\left\{\begin{array}{l}
\lambda_{n} \\
C_{n} \\
E_{n} \\
S_{n}
\end{array}\right.
$$

where System $_{n}$ is the index of the system (for $n=1, \ldots, N$ ), $\lambda_{n}$ is the failure frequency per hour during the year, $C_{n}$ is the purchase and implementation cost of the newtype type, $E_{n}$ is the environment factor and $S_{n}$ is the safety factor. 
The aim of our work is to define a replacement policy for these obsolete systems in the context of dependability and to help the decision maker find an optimal strategy among them. We identify the optimal systems to be replaced by the newtype (challenger) but with these considerations: budget, optimal benefit from the residual lifetime of the old-type, and the environment/safety factors.

\subsection{Genetic Algorithms Approach and Problem Formulation}

To solve our optimization problem, we propose to develop an approach using a genetic algorithm (GA).

Genetic algorithms (GAs) are powerful bio-inspired algorithms that have been successfully used in several research problems: permutation flow shop [16], correcting the fine structure of surfaces [17], the synthesis of production-control systems [18], etc. The GA belongs to the soft computing technologies, who owe their name to their operational similarities with the biological and behavioral phenomena of living beings. Their primary target is the optimization of an assigned objective function (fitness).

GA was originally developed by Holland [19]. In general, genetic algorithms are based on the following steps [20]:

(1) Creation of a random initial population of potential solutions to the problem and evaluation of these individuals in terms of their fitness, i.e. of their corresponding objective function values;

(2) Selection of a pair of individuals as parents;

(3) Crossover of the parents, with the generation of two children;

(4) Replacement in the population, so as to maintain a constant population number;

(5) Genetic mutation;

(6) Repeat steps until satisfying solution is obtained.

The maximizing objective function (fitness) of our problem could be written as:

$$
\text { fitness }=\operatorname{Max} \sum_{n=1}^{N}\left[\lambda_{n}+\frac{1}{C_{n}}+\frac{1}{E_{n}}+\frac{1}{S_{n}}\right]
$$

The fitness (7) is under the following constraint:

$$
\sum_{n=1}^{N} C_{n} \leq \text { Budget }
$$

The objective function (7) allows for identifying the optimal systems (high failure frequency, more polluting, less secure and lowest purchase cost of the challenger) recommended for the replacement by the new-type units. The solution is given under the constraint of the budget (8). 
A binary coding is the most suitable in this case. The number of genes per individual (chromosome) is equal to the number of systems in order of appearance. Therefore, the size of the chromosome must be equal to $N$. Figure 2 shows an example of an eventual individual. A random initial population of potential solutions is given. If the system is marked in the individual, then it is assigned the value 1, otherwise 0 . All systems which are assigned the value 1, their parameters will be implemented and evaluated in the fitness. A fixed number of iterations is stated after different times of run. During generations, our genetic algorithm seeks the optimal solution until convergence and stopping criterion.

\begin{tabular}{rl|l|l|l|l|l|l|l|}
\hline 0 & 1 & 1 & 1 & 0 & $\ldots \ldots \ldots \ldots \ldots$ & 1 & 1 \\
System $_{n}:$ & 1 & 2 & 3 & 4 & 5 & $\ldots \ldots \ldots \ldots \ldots$ & $N-1$ & $N$
\end{tabular}

Figure 2

Example of an individual

In the individual shown in Fig. 2, the eventual solution is given by the replacement

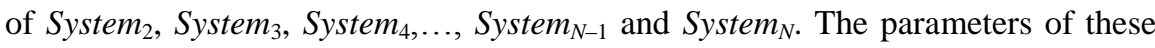
systems will be implemented and evaluated by the fitness.

Table 1

Comparison between main features of previous models and our approach

\begin{tabular}{|c|c|c|c|c|}
\hline References & Context & $\begin{array}{c}\text { Number of } \\
\text { industrial } \\
\text { systems }\end{array}$ & $\begin{array}{l}\text { Environment and } \\
\text { safety factors of } \\
\text { the old-type units }\end{array}$ & $\begin{array}{l}\text { Transition } \\
\text { strategy }\end{array}$ \\
\hline$[5]$ & economics & 1 & - & $\begin{array}{l}\text { replacement at } \\
\text { fixed intervals }\end{array}$ \\
\hline [7] & economics & 1 & - & $\begin{array}{l}\text { replacement at } \\
\text { fixed intervals }\end{array}$ \\
\hline [6] & economics & 1 & - & $\begin{array}{l}\text { replacement at } \\
\text { fixed intervals }\end{array}$ \\
\hline [8] & Engineering & 1 & - & $\begin{array}{l}\text { replacement at the } \\
\text { first failure }\end{array}$ \\
\hline [13] & Engineering & $N$ identical & - & $K$ strategy \\
\hline [9] & Engineering & $N$ identical & - & $K$ strategy \\
\hline [14] & Engineering & $N$ identical & - & $K$ strategy \\
\hline$[10,15]$ & Engineering & $N$ identical & - & $K$ strategy \\
\hline $\begin{array}{l}\text { [our model in } \\
\text { this paper] }\end{array}$ & Engineering & $N$ different & $E_{n}, S_{n}$ & $\begin{array}{l}\text { at the end of the } \\
\text { year, according to } \\
\text { the budget and the } \\
\text { selection is made } \\
\text { using genetic } \\
\text { algorithms }\end{array}$ \\
\hline
\end{tabular}


After each generation, a new solution is given by the algorithm. The fitness function evaluates these solutions and they are ranked. This ranking is used in the selection procedure (standard roulette), which is performed in such a way that in the long run the best individuals will have a greater probability to be selected as parents, in resemblance to the natural principles of the "survival of the fittest". Similarly, the ranking is used in the replacement procedures to decide who among the parents and the daughters should survive in the next population. An algorithm based on these procedures is often referred to as a steady-state GA [20].

We assume that there are a few random solutions beyond the budget; they will be kept to maintain diversity and to avoid stalling at local optima.

Table 1 summarizes the main features of different previous works and our proposition in this paper.

\subsection{Case Study}

In this subsection, we present a numerical application of our model. The case considered here is a set of $(N=14)$ different industrial systems subject to technological change (obsolescence). The data of these systems are reported in Table 2 and $\left(\right.$ Budget $\left.=50 \times 10^{2} \$\right)$.

Table 2

Data of the systems

\begin{tabular}{|c|c|c|c|c|}
\hline System $_{n}$ & $\begin{array}{c}\text { Failure } \\
\text { frequency } \lambda_{n} \\
\text { (hour }{ }^{-1} \text {, during } \\
\text { the year) } \times 10^{-6}\end{array}$ & $\begin{array}{l}\text { Purchase and } \\
\text { implementation } \\
\text { cost of the } \\
\text { challenger } C_{n} \\
\left(10^{2} \$\right)\end{array}$ & Safety factor $S_{n}$ & $\begin{array}{l}\text { Environment } \\
\text { factor } E_{n}\end{array}$ \\
\hline 1 & 3.54 & 6.40 & 0.92 & 0.50 \\
\hline 2 & 2.26 & 8.20 & 0.95 & 0.77 \\
\hline 3 & 5.37 & 6.00 & 0.84 & 0.80 \\
\hline 4 & 4.88 & 3.80 & 0.87 & 0.65 \\
\hline 5 & 4.66 & 5.21 & 0.91 & 0.54 \\
\hline 6 & 2.28 & 3.01 & 0.86 & 0.66 \\
\hline 7 & 8.01 & 7.80 & 0.80 & 0.71 \\
\hline 8 & 6.01 & 6.50 & 0.85 & 0.59 \\
\hline 9 & 7.87 & 8.40 & 0.93 & 0.82 \\
\hline 10 & 6.07 & 6.05 & 0.91 & 0.85 \\
\hline 11 & 5.90 & 4.33 & 0.81 & 0.79 \\
\hline 12 & 4.26 & 3.41 & 0.79 & 0.85 \\
\hline 13 & 6.87 & 5.00 & 0.83 & 0.90 \\
\hline 14 & 3.40 & 7.00 & 0.96 & 0.87 \\
\hline
\end{tabular}




\subsubsection{Problem Formulation}

The fitness function of this case study can then be written as follows:

$$
\text { fitness }=\operatorname{Max} \sum_{n=1}^{14}\left[10^{4} \times \lambda_{n}+10 \times \frac{1}{C_{n}}+10^{-2} \times \frac{1}{E_{n}}+10^{-2} \times \frac{1}{S_{n}}\right]
$$

where the weighting $10^{4}, 10$ and $10^{-2}$ are introduced to balance the fitness. This objective function tries to maximize the number of industrial systems which should be replaced by new-type units. The unidentified systems their residual lifetime will be exploited.

The fitness function (9) is subject to:

$$
\sum_{n=1}^{N=14} C_{n} \leq 50 \times 10^{2}
$$

Table 2 contains the parameters related to the systems, whereas Table 3 contains the rules and the parameters for the GA implemented in order to solve the objective optimization problem. The values of the parameters were chosen after times of run to achieve a convergence.

Table 3

Genetic algorithm rules and parameters

\begin{tabular}{|c|c|}
\hline GA property & Value \\
\hline Number of genes for individual & 14 \\
\hline Number of individuals (population size) & 60 \\
\hline Number of generations (termination criterion) & 500 \\
\hline Mutation probability & 0.001 \\
\hline Selection technique & Standard Roulette \\
\hline
\end{tabular}

\subsubsection{Results and Discussion}

The results of the GA optimization process and the convergence of the fitness are shown in Fig. 3.

We remark that the convergence of the proposed algorithm is at 100 generations (see Fig. 3). The number of systems and their parameters has been controlled by the algorithm. As mentioned in Subsection 3.1, we assume that few random solutions exist beyond the budget, and they will be introduced during iterations to maintain diversity; hence, we obtained fitness values greater than the convergence value. 


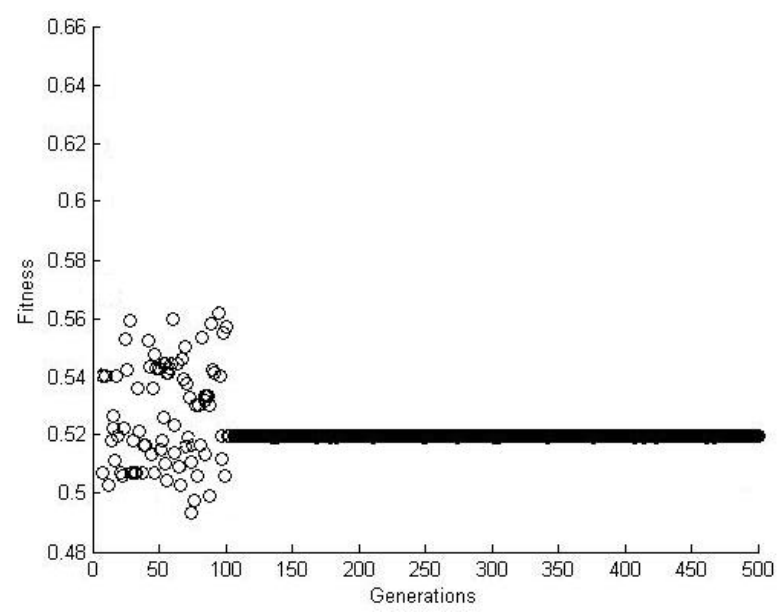

Figure 3

Result of the GA process for the optimal replacement strategy

The solution is identified in the individual represented in Fig. 4. All the systems assigned the value 1 are considered optimal for the replacement by the newtechnology units.

\begin{tabular}{|l|l|l|l|l|l|l|l|l|l|l|l|l|l|}
\hline 0 & 0 & 1 & 1 & 0 & 1 & 1 & 0 & 1 & 0 & 1 & 1 & 1 & 0 \\
\hline
\end{tabular}

Figure 4

Individual of the solution

Table 4 illustrates the systems identified in the individual (see Fig. 4) of the optimal solution.

Table 4

Systems recommended for the replacement policy

\begin{tabular}{|c|c|}
\hline \multicolumn{2}{|c|}{ System $_{n}$} \\
\hline System $_{3}$ & System $_{9}$ \\
\hline System $_{4}$ & System $_{11}$ \\
\hline System $_{6}$ & System $_{12}$ \\
\hline System $_{7}$ & System $_{13}$ \\
\hline
\end{tabular}

\section{Conclusions}

In this paper we proposed a model to deal with the obsolescence of industrial systems in the context of engineering. A genetic algorithm was elaborated for solving a case of several systems subject to technological obsolescence. The model was illustrated on a case study under the following considerations: environment/safety factors, failure frequencies of the old-types units and the purchase/implementation cost of the new-type units (challengers). 
A major advantage of this model consists of the possibility to find an optimal replacement policy when we have a complex case study with many systems and several parameters. The difficulty persists in the choice of the parameter values of the algorithm and the program development.

Future work will need to define an optimal policy in the case of dependent systems, the impact of the new-challengers on the installation, and comparative results using other optimization methods.

\section{References}

[1] M. A. Mellal, S. Adjerid, D. Benazzouz: Modeling and Simulation of Mechatronic System to Integrated Design of Supervision: Using a Bond Graph Approach, Applied Mechanics and Materials, Vol. 86, pp. 467-470, 2011

[2] A. R. Conn, L. A. Deleris, J. R. M. Hosking, T. A. Thorstensen: A Simulation Model for Improving the Maintenance of High Cost Systems With Application to an Offshore Oil Installation, Quality and Reliability Engineering International, Vol. 26, No. 7, pp. 733-748, 2010

[3] M. A. Mellal, S. Adjerid, D. Benazzouz: Modeling and Simulation of Mechatronic System to Integrated Design of Supervision: Using a Bond Graph Approach, in Proceedings of ECMS'2011 European Council on Modelling and Simulation, Krakow, Poland, pp. 370-373, 2011

[4] E. P. Zafiropoulos, E. N. Dialynas: Reliability and Cost Optimization of Electronic Devices Considering the Component Failure Rate Uncertainty, Reliability Engineering and System Safety, Vol. 84, No. 3, pp. 271-284, 2004

[5] E. J. Elton, M. J. Gruber: On the Optimally of an Equal Life Policy for Equipment Subject to Technological Improvement, Operational Research, Vol. 27, pp. 93-99, 1976

[6] I. E. Schochetman, R. L. Smith: Infinite Horizon Optimality Criteria for Equipment Replacement Under Technological Change, Operations Research Letters, Vol. 35, No. 4, pp. 485-492, 2007

[7] N. Hritonenko, Y. Yatsenko: Optimal Equipment Replacement Without Paradoxes: A Continous Analysis, Operations Research Letters, Vol. 35, No. 2, pp. 245-250, 2007

[8] E. Borgonovo, M. Marseguerra, E. Zio: A Monte Carlo Methodological Approach to Plant Availability Modeling with Maintenance-aging and Obsolescence, Reliability Engineering and System Safety, Vol. 67, No. 1, pp. $61-73,2000$

[9] S. Mercier, P. E. Labeau: Optimal Replacement Policy for a Series System with Obsolescence, Applied Stochastic Models in Business and Industry, Vol. 20, No. 1, pp. 73-91, 2004 
[10] J. Clavareau, P. E. Labeau: Maintenance and Replacement Policies Under Technological Obsolescence, Reliability Engineering and System Safety, Vol. 94, No. 2, pp. 370-381, 2009

[11] P. K. Nguyen Thi, T. G. Yeung, B. Castanier: Optimal Maintenance and Replacement Decisions Under Technological Change, in Proceedings of ESREL'2010: European Safety and Reliability Conference, Rhodes, Greece, 2010

[12] O. Michel, P. E. Labeau, S. Mercier: Monte Carlo Optimization of the Replacement Strategy of Components Subject to Technological Obsolescence, in Proceedings of the International Conference on Probabilistic Safety Assessment and Management, Berlin, Germany, 2004

[13] D. Elmakis, G. Leitin, A. Lisnianski: Optimal Scheduling for Replacement of Power System Equipment with New-type One, in Proceedings of the $3^{\text {rd }}$ International Conference on Mathematical Methods in Reliability, Trondheim, Norway, 2002

[14] S. Mercier: Optimal Replacement Policy for Obsolete Components with General Failure Rates, Applied Stochastic Models in Business and Industry- Reliability, Vol. 24, No. 3, pp. 221-235, 2008

[15] J. Clavareau, P. E. Labeau: A Petri Net-based Modelling of Replacement Strategies Under Technological Obsolescence, Reliability Engineering and System Safety, Vol. 94, No. 2, pp. 357-369, 2009

[16] K. Balazs, Z. Horvath, L. T. Koczy: Different Chromosome-based Evolutionary Approaches for the Permutation Flow Shop Problem, Acta Polytechnica Hungarica, Vol. 9, No. 2, pp. 115-138, 2012

[17] G. Gyurecz, G. Renner: Correcting Fine Structure of Surfaces by Genetic Algorithm, Acta Polytechnica Hungarica, Vol. 8, No. 6, pp. 181-190, 2011

[18] P. Y. Mok: Genetic Synthesis of Production-control Systems for Unreliable Manufacturing Systems with Variable Demands, Computers and Industrial Engineering, Vol. 61, No. 1, pp. 198-208, 2011

[19] J. H. Holland: Adaptation in Natural and Artificial Systems, Ann Arbor, MI: University of Michigan Press, USA, 1975

[20] S. Sumathi, P. Surekha: Computational Intelligence Paradigms, Taylor \& Francis Group, London, United Kingdom, 12-15, 2010 